\title{
Conductive Nanoparticles in Dielectrics: A Comparative Study
}

\author{
Agamyrat Agambayev, Mohamed Farhat, Hakan Bagci, and Khaled N. Salama \\ Division of Computer, Electrical, and Mathematical Sciences and Engineering \\ King Abdullah University of Science and Technology (KAUST) \\ Thuwal 23955-6900, Saudi Arabia \\ \{agamyrat.agambayev, mohamed.farhat, hakan.bagci, khaled.salama\}@kaust.edu.sa
}

\begin{abstract}
The Maxwell-Garnett method is used to predict the effective dielectric constant and the tangent loss of various composites consisting of a PVDF-TrFE-CFE-matrix and conductive microsphere fillers made of $\mathrm{Cu}, \mathrm{Ni}, \mathrm{W}, \mathrm{Zn}$, or $\mathrm{Fe}$. Simulation results demonstrate that for small filler fraction values and at low frequencies, the electrical properties of the resulting composite do not depend on the conductivity of the filler. These findings show that composites fabricated using cheaper metal nanoparticle fillers are as effective as those fabricated using expensive ones.
\end{abstract}

Keywords- Composite materials, conductive nanoparticles, Maxwell-Garnett mixing rule.

\section{INTRODUCTION}

A capacitor's energy storage capacity depends on its size as well as the dielectric permittivity of the spacer/filling material used [1-5]. In recent years, due to the advancements in fabrication technologies, the size of electronic systems and their individual components have significantly shrunk. Consequently, to obtain adequate levels of energy storage, materials with high permittivity values, commonly refered to as high $\mathrm{K}$, have to be used [2-6]. One way of fabricating such materials is through reinforcing dielectrics, such as polymers, with highly conductive metallic fillers (Fig.1). In addition to high permittivity, these composite have several desired mechanical properties, which has lead to their popular use in embedded circuits, e.g. in flexible capacitors [6-11].

In this work, electrical properties of metal-dielectric composites are predicted using the Maxwell-Garnet method [12]. More specifically, the effective dielectric constant and the tangent loss of various composites consisting of a PVDF-TrFECFE-matrix and conductive microsphere fillers made of $\mathrm{Cu}$, $\mathrm{Ni}, \mathrm{W}, \mathrm{Zn}$, or $\mathrm{Fe}$ are computed in the dilute regime (before percolation regime start). Comparison of the predicted values show that at low frequencies, electrical properties of the composites are same for different type fillers as long as their shape and size and the filler fraction of the mixing are the same. In other words, the under these conditions, the conductivity of the fillers does not have any affect on the permittivity and tangent loss of the resulting composite.

These findings might help with fabrication procedures in determining the "best" type of conductive filler when other factors such as the cost of metals need to be considered.

The authors would like to thank the Saudi Basic Industries Corporation (SABIC) for partially funding this work under Grant No. RGC/3/2385-01

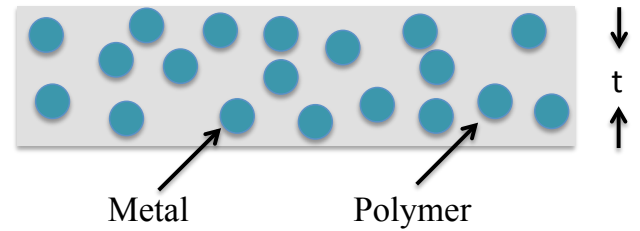

Fig.1. Representation of a polymer composite with spherical fillers.

\section{Method AND MAterials}

The permittivity of a dielectric can be mathematically modeled by [13]

$$
\varepsilon_{e}=\varepsilon_{\infty, e}+\frac{\varepsilon_{s, e}-\varepsilon_{\infty, e}}{\left(1+(j \omega \tau)^{1-\alpha}\right)^{\beta}}
$$

where $\varepsilon_{s, e}$ and $\varepsilon_{\infty, e}$ are static and high frequency (optical) relative permittivities, $\alpha$ and $\beta$ are Cole-Cole and ColeDavidson constants, and $\tau$ is the relaxation time. Similarly, the permittivity of a conductor is given by [13]

$$
\varepsilon_{i}=\varepsilon_{\infty, i}+\frac{\sigma}{j \omega \varepsilon_{0}}
$$

where $\varepsilon_{\infty, i}$ is the optical permittivity (for "perfect" metals, such as Copper, $\left.\varepsilon_{\infty, i}=1\right), \quad \sigma$ is the conductivity, and $\varepsilon_{0}=8.85 \times 10^{-12} \mathrm{~F} / \mathrm{m}$ is the permittivity of the vacuum. At low frequencies, the imaginary part of the permittivity is dominant.

The Maxwell-Garnett formula is used to obtain an analytical expression for the effective permittivity of a composite with a dielectric matrix and spherical conductive fillers:

$$
\varepsilon_{e f f}=\varepsilon_{e}+3 \varepsilon_{e}\left(\frac{F F\left(\varepsilon_{i}-\varepsilon_{e}\right)}{\varepsilon_{i}+2 \varepsilon_{e}-F F\left(\varepsilon_{i}-\varepsilon_{e}\right)}\right) .
$$

Here, $\varepsilon_{e}$ [see (1)] and $\varepsilon_{i}$ [see (2)] are permittivities of matrix/host and filler materials, and $F F$ is the volume fraction of the fillers to the overall volume of the composite.

\section{RESULTS AND DISCUSSION}

The parameters used in (1) for the PVDF-TrFE-CFE matrix are $\alpha=0.45, \beta=0, \varepsilon_{s, e}=50, \varepsilon_{\infty, e}=4$, and $\tau=1.75 \times 10^{-6} \mathrm{~s}$ and the conductivities of the filler microspheres made of $\mathrm{Cu}$, $\mathrm{W}, \mathrm{Zn}, \mathrm{Ni}$, or Fe are given in Table I. Diameters of all fillers are $800 \mathrm{~nm}$. 
TABLE I. CONDUCTIVITIES OF FILLER MATERIALS

\begin{tabular}{|c|c|c|c|c|c|}
\hline Filler & $\mathrm{Cu}$ & $\mathrm{W}$ & $\mathrm{Zn}$ & $\mathrm{Ni}$ & $\mathrm{Fe}$ \\
\hline$\sigma[M S / m]$ & 59.6 & 17.9 & 16.9 & 14.3 & 10.1 \\
\hline
\end{tabular}

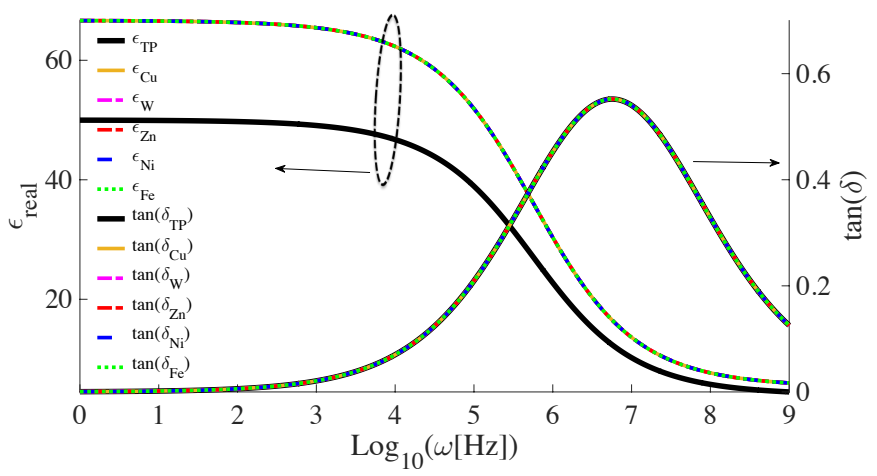

Fig. 2. Complex permittivity of the composites with different type fillers within the frequency band $1 \mathrm{~Hz}$ and $1 \mathrm{GHz}$. Filler fraction $\mathrm{FF}=0.10$

For the first simulation, filler fraction $\mathrm{FF}=0.10$ while the frequency is varied from $1 \mathrm{~Hz}$ to $1 \mathrm{GHz}$. The effective permittivity of the composites is computed using (3) at every frequency sample. Fig. 2 plots the real part of the permittivity and the tangent loss of PVDF-TrFE-CFE and each composite versus frequency. The results clearly show that the conductivity of the metal fillers does not have any affect on the electrical properties of the resulting composite for a rather broadband of frequencies. This result can be explained as follows. The permittivity of the metallic fillers is significantly larger that of the polymer $\left(\left|\varepsilon_{i} / \varepsilon_{e}\right| \gg 1\right)$. Consequently, the ratio $\left|\varepsilon_{i} / \varepsilon_{e}\right|$ used in the Maxwell-Garnett rule (3) dominates all other terms when the frequency is low enough. This makes the effective permittivity a function of only the filling fraction FF.

For the second simulation, frequency is kept constant at 10 $\mathrm{KHz}$ while filler fraction $\mathrm{FF}$ is varied from 0 to 0.20 . Fig. 3 plots the real part of the permittivity and the tangent loss of each composite versus filler factor FF. These results also agree with the explanation above. Effective electrical properties of the composites vary only with the filler fraction and do not depend on the conductivity of the fillers. The results in Figs. 2 and 3 suggest that $\mathrm{Fe}$ can effectively replace (the more expensive) $\mathrm{Cu}$ as a filler without affecting the electrical properties of the resulting composite even though the conductivity of $\mathrm{Cu}$ is six times greater.

\section{CONLCUSION}

The effect of metal filler's conductivity on the electrical properties of polymer-metal composites is numerically characterized using the Maxwell-Garnet method. Simulation results show that, for small values of filler fraction and at low frequencies, electrical properties of the polymer-metal composites do not depend on the conductivity of metal filler.

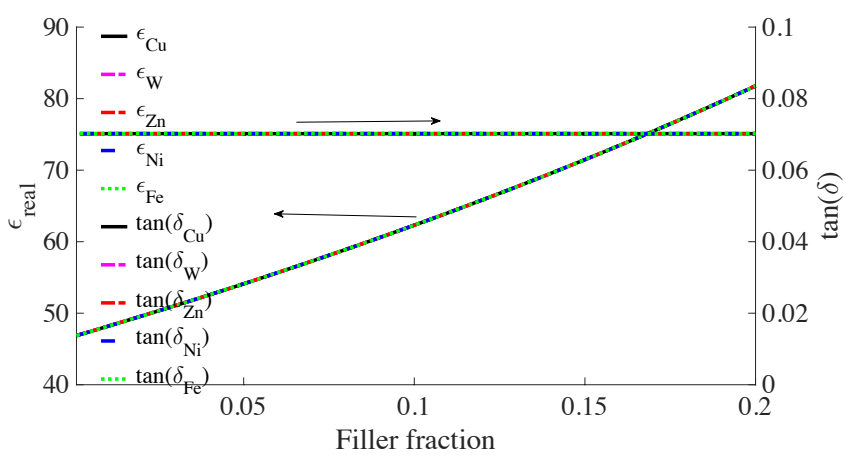

Fig.3. Complex permittivity of the composites for filler fraction changing between 0 and 0.20 at $10 \mathrm{kHz}$.

\section{REFERENCES}

[1] V. K Thakur and M. R. Kessler, "Polymer nanocomposites: New advanced dielectric materials for energy storage applications," Adv. Mater., pp. 207-257, Jan. 2014.

[2] J. Lu and C. Wong, "Recent advances in high-k nanocomposite materials for embedded capacitor applications," IEEE Trans. Dielectr. Electr. Insul., vol. 15, pp. 1322-1328, Oct. 2008.

[3] P. Barber et al., "Polymer composite and nanocomposite dielectric materials for pulse power energy storage," Materials, vol. 2, pp. 1697 1733, Oct. 2009.

[4] E. R. R. Tummala and E. J. Rymaszewski, Microelectronics packaging Handbook. Cambridge Univ Press, 1997.

[5] H. Gargama, A. Thakur, and S. Chaturvedi, "Polyvinylidene fluoride/nickel composite materials for charge storing, electromagnetic interference absorption, and shielding applications," J. Appl. Phys, vol. 117 , pp. 224903, June 2015.

[6] L. Zhang and Z.-Y. Cheng, "Development of polymer-based 0-3 composites with high dielectric constant," J. Adv. Dielect., vol. 1, pp. 389-406, Oct. 2011.

[7] Z.-M. Dang, et al., "Fundamentals, processes and applications of highpermittivity polymer-matrix composites," Prog. Mater. Sci, vol. 57, pp. 660-723, Aug. 2012.

[8] Z.-M. Dang, Y. H. Lin, and C. W. Nan, "Novel ferroelectric polymer composites with high dielectric constants," Adv. Mater., vol. 15, pp. 1625-1629, Oct. 2003.

[9] S. Stankovich, et al. "Graphene-based composite materials," Nature, vol. 442, pp. 282-286, July 2006.

[10] H. He, S. Lau, H. L. Cha, and J. Fan, "High dielectric permittivity and low percolation threshold in nanocomposites based on poly (vinylidene fluoride) and exfoliated graphite nanoplates," Adv. Mater., vol. 21, no. 6, pp. 710-715, Feb. 2009.

[11] M. Panda, V. Srinivas, and A. Thakur, "Role of polymer matrix in large enhancement of dielectric constant in polymer-metal composites," Appl. Phys. Lett., vol. 99, pp. 042905, 2011.

[12] M. J. Garnett, "Colours in Metal Glasses and in Metallic Films," in Proc. R. Soc. London A Math. Phys. Eng. Sci., pp. 443-445, 1904.

[13] S. Havriliak and S. Negami, "A complex plane analysis of alphadispersion in some polymer systems" in Proc. J. Polym. Sci. Pt. CPolym. Symp., vol. 14, pp 99-11,1966. 